\title{
A New Approach for Exponential Stability Criteria of New Certain Nonlinear Neutral Differential Equations with Mixed Time-Varying Delays
}

\author{
Janejira Tranthi ${ }^{1}$, Thongchai Botmart ${ }^{1}$, Wajaree Weera ${ }^{2, *}$ and Piyapong Niamsup ${ }^{3}$ \\ 1 Department of Mathematics, Faculty of Science, Khon Kaen University, Khon Kaen 40002, Thailand \\ 2 Department of Mathematics, Faculty of Science, University of Phayao, Phayao 56000, Thailand \\ 3 Department of Mathematics, Faculty of Science, Chiang Mai University, Chiang Mai 50200, Thailand \\ * Correspondence: wajaree.we@up.ac.th
}

Received: 15 July 2019; Accepted: 9 August 2019; Published: 12 August 2019

\begin{abstract}
This work is concerned with the delay-dependent criteria for exponential stability analysis of neutral differential equation with a more generally interval-distributed and discrete time-varying delays. By using a novel Lyapunov-Krasovkii functional, descriptor model transformation, utilization of the Newton-Leibniz formula, and the zero equation, the criteria for exponential stability are in the form of linear matrix inequalities (LMIs). Finally, we present the effectiveness of the theoretical results in numerical examples to show less conservative conditions than the others in the literature.
\end{abstract}

Keywords: exponential stability; certain nonlinear neutral differential equations; mixed time-varying delays

\section{Introduction}

Many scientists have worked on the stability criteria for the problem of neutral delayed differential equation (NDE) with constant delays [1-9] as follows:

$$
\frac{d}{d t}[x(t)+p x(t-\tau)]+a x(t)-b \tanh x(t-\sigma)=0, \quad t \geq 0,
$$

where $|p|<1$ and $a, \tau, b, \sigma$ are real positive constants. With each solution $x(t)$ of (1), the initial condition is satisfied:

$$
x(s)=\phi(s), \quad s \in[-r, 0], \quad \text { where } \phi \in C([-r, 0], R), r=\max \{\tau, \sigma\} .
$$

The NDE (1) as a special case has been considered by many researchers for the study of the dynamical characteristics of the neural networks of the Hopfield type; see [10-13] and the references cited therein. As we all know, a small change in delay may destabilize a system [3,5-7,9]. Hence, many researchers increased their study of the stabilization of the system by proposing the criteria in several forms.

In [6], the delay-dependent stability criterion of certain neutral differential equations was considered by the integral inequalities approaches. However, the sufficient condition only depends on the discrete delay $\sigma$ and does not depend on the neutral delay $\tau$. An improved stability criterion for a class of neutral differential equations was studied in [8] by introducing a new Lyapunov functional and avoiding the use of the stability assumption on the main operators. In [1], the authors considered using a Lyapunov-Krasovskii functional technique and a descriptor model transformation approach to transform the system with distributed and discrete delays. The issue of exponential stability analysis 
of the neutral differential equation as in (1) has been presented $[4,5]$ by utilizing the descriptor form, Lyapunov-Krasovskii functionals, and model transformation.

In the past few years, many researches have studied several methods in time-varying delay,s which can be considered as closer way to reflect the realistic situation because the time delays are usually not constant. In $[1,14,15]$, the authors considered the problem of NDE for exponential stability with time-varying delays as follows:

$$
\frac{d}{d t}[x(t)+p x(t-\tau(t))]+a x(t)-b \tanh x(t-\sigma(t))=0, \quad t \geq 0,
$$

where $|p|<1$ and $a, b$ are real positive constants and $\sigma(t)$ and $\tau(t)$ are discrete and neutral time-varying delays, respectively,

$$
\begin{array}{ll}
0 \leq \tau(t) \leq \tau, & \dot{\tau}(t)<\tau_{d}, \\
0 \leq \sigma(t) \leq \sigma, & \dot{\sigma}(t)<\sigma_{d},
\end{array}
$$

where $\tau$ and $\sigma$ are given real positive constants. For each solution $x(t)$ of (2), the initial condition is satisfied:

$$
x_{0}(t)=\phi(t), \quad \text { where } \phi \in C([-r, 0], R), \quad r=\max \{\tau, \sigma\} .
$$

In [14], the sufficient conditions were presented with the use of descriptor form, Lyapunov functions, the Leibniz-Newton formula, and radially-unbounded function.

On the other hand, distributed delays, as one of the most common types, have received considerable attention in recent years. In a practical system, the distributed delay is often useful in modeling processes that are (i) described by flow rates (aggregates) of entities that move at different rates through the given irreversible process (ii). Recently, a certain neutral differential equation with distributed and discrete time-varying delays was considered in [16] as follows:

$$
\frac{d}{d t}[x(t)+p x(t-\tau(t))]+a x(t)-b \tanh x(t-\sigma(t))-c \int_{t-\rho(t)}^{t} x(s) d s=0, t \geq 0,
$$

where $|p|<1$ and $a, b, c$ are real positive constants. $\sigma(t), \rho(t)$, and $\tau(t)$ are discrete, distributed, and neutral time-varying delays, respectively.

In practice, distributed time delays arise in models for a variety of applications including population dynamic [17], the predator-prey model [18,19], the neuronal model [20,21], neural networks [22-25], and a nonlinear system [26], which considered the distributed delay of the activation function [24]. From the discussions above, there still exists room for further improvement in NDE, and it might occur that there are connection between the distributed time-varying delay and $\tanh x(s)$. As a result, it is more natural to consider NDE with the distributed delay of $\tanh x(s)$. However, in recent works, there were no models $[1-9,14,15,27-30]$ that include distributed time-varying delays.

Our main contributions can be summarized as the following aspects. First, our work is the first attempt to study for the problem of new certain nonlinear neutral differential equation for exponential stability with a more generally interval-distributed and discrete time-varying delays. The Lyapunov-Krasovkii functional, descriptor model transformation, the Newton-Leibniz formula, and utilization of the zero equation are applied. The exponential stability criteria are in the form of linear matrix inequalities (LMIs). Second, note that most of the existing NDE results $[1,9,14-16]$ cannot be applied to the situation when there are two distributed time-varying delays. Finally, a comparison of the maximum delay bounds obtained by our proposed methods with those in many existing literature works $[1,9,14,15]$ is conducted, which supports that the exponential stability criteria provided in this paper can enhance the feasible region effectively and significantly. 
The rest of this paper is arranged as follows. Section 2 shows the definitions, notations, and lemmas for applying the main results of the paper. The exponential stability criteria for delay-dependent sufficient conditions are shown in Section 3. Numerical examples presented for effectiveness obtained consequences that are manifested in Section 4. This work ends with the conclusion in Section 5 .

Notation: The following notations will be used throughout this manuscript. $B^{T}$ denotes the transpose of matrix $B ; B \geq 0(B>0)$ means that $B$ is semi-positive definite (positive definite); $\|\cdot\|$ denotes the Euclidean norm of the vector or matrix, $\|x\|_{s}=\sup _{-r \leq s \leq 0}\|x(s)\|$ and $\|\dot{x}\|_{l}=\sup _{-r \leq s \leq 0}\|\dot{x}(s)\|_{l}$. The symbol $*$ stands for the transposed elements in the symmetric positions.

\section{Problem Formulation and Preliminary}

Consider the neutral differential equation with time-varying delays as follows:

$$
\frac{d}{d t}[x(t)+p x(t-\tau(t))]+a x(t)-b \tanh x(t-\sigma(t))-e \int_{t-h(t)}^{t} x(s) d s-f \int_{t-l(t)}^{t} \tanh x(s) d s=0,
$$

where $|p|<1$ and $a, b, e, f$ are real positive constants. $h(t), l(t)$, and $\sigma(t)$ are distributed and discrete interval time-varying delays, and $\tau(t)$ is a neutral interval time-varying delay, satisfying,

$$
\begin{aligned}
& 0 \leq \tau_{1} \leq \tau(t) \leq \tau_{2}, \quad \dot{\tau}(t) \leq \tau_{d} \\
& 0 \leq \sigma_{1} \leq \sigma(t) \leq \sigma_{2}, \quad \dot{\sigma}(t) \leq \sigma_{d} \\
& 0 \leq h_{1} \leq h(t) \leq h_{2} \\
& 0 \leq l_{1} \leq l(t) \leq l_{2}
\end{aligned}
$$

where $\tau_{1}, \tau_{2}, \sigma_{1}, \sigma_{2}, h_{1}, h_{2}, l_{1}, l_{2}, \tau_{d}$, and $\sigma_{d}$ are given positive real constants. For each solution $x(t)$ of (4), the initial condition is satisfied:

$$
x_{0}(t)=\phi(t), \quad t \in[-r, 0]
$$

where $\phi \in C([-r, 0] ; R)$ and $r=\max \left\{\tau_{2}, \sigma_{2}, h_{2}, l_{2}\right\}$.

Definition 1 ([14]). If there exist real positive constants $\lambda, K$ such that:

$$
\|x(t)\| \leq K e^{-\lambda t} \sup _{-r \leq s \leq 0}\|x(s)\|=K e^{-\lambda t}\|x\|_{s}
$$

then the equilibrium point of Equation (2) is exponentially stable.

Lemma 1. (Jensen's inequality [31]). For positive real number $h$, any symmetric positive definite matrix $Q$ and vector function $\dot{x}(t):[-h, 0] \rightarrow R^{n}$ such that the following inequality holds:

$$
-h \int_{-h}^{0} \dot{x}^{T}(s+t) Q \dot{x}(s+t) d s+\left(\int_{-h}^{0} \dot{x}(s+t) d s\right)^{T} Q\left(\int_{-h}^{0} \dot{x}(s+t) d s\right) \leq 0 .
$$

Lemma 2. (Cauchy's inequality [31]). If there exists constant symmetric positive definite matrix $P \in R^{n \times n}$ and $a, b \in R^{n}$, we have:

$$
\pm 2 a^{T} b \leq a^{T} P a+b^{T} P^{-1} b
$$


Lemma 3 ([16]). For any constant $Q \in R^{n \times n}$ is a symmetric positive definite matrix, $h(t)$ is a discrete time-varying delay with (7), and vector function $\omega:\left[-h_{2}, 0\right] \rightarrow R^{n}$ such that the following inequality holds:

$$
\begin{aligned}
& -\left[h_{2}-h_{1}\right] \int_{-h_{2}}^{-h_{1}} \omega^{T}(s) Q \omega(s) d s \\
\leq & -\int_{-h(t)}^{-h_{1}} \omega^{T}(s) d s Q \int_{-h(t)}^{-h_{1}} \omega(s) d s-\int_{-h_{2}}^{-h(t)} \omega^{T}(s) d s Q \int_{-h_{2}}^{-h(t)} \omega(s) d s .
\end{aligned}
$$

Lemma 4. (Peng-Park's integral inequality [32]). For positive scalars $\tau$ and $\tau(t)$ satisfying $0<\tau(t)<\tau$, vector function $\dot{x}(s):[-\tau, 0] \rightarrow R^{n}$, any matrix $Z \in \mathbb{R}^{n \times n}, Z=Z^{T}$, matrix $S \in \mathbb{R}^{n \times n}$, and any matrix $\left[\begin{array}{ll}Z & S \\ * & Z\end{array}\right] \geq 0$ such that the following concerned inequality holds:

$$
-\tau \int_{t-\tau}^{t} \dot{x}^{T}(s) Z \dot{x}(s) d s \leq \omega^{T}(t) \ominus \omega(t)
$$

where $\omega=\left[x^{T}(t), x^{T}(t-\tau(t)), x^{T}(t-\tau)\right]$ and $\ominus=\left[\begin{array}{ccc}-Z & Z-S & S \\ * & 2 Z+S+S^{T} & Z-S \\ * & * & -Z\end{array}\right]$.

Remark 1. It should be pointed out that the problem of the delay differential equation with distributed delay function of $\tanh x(s)$ is more general than [16], where the delay is not considered. Generally applicable nonlinear system contain with mixed time-varying delays, for instance the population dynamics and predator-prey model that were studied in [17-19]. It should be noted that this is the first encountered when the distributed time-varying delay function of $\tanh x(s)$ is studied, which includes both distributed and discrete delays for the system.

\section{Stability Analysis}

For this part, we analyze the issue of the exponential stability criteria for the neutral differential Equation (4) with time-varying delays. From the model transformation technique, we get the Newton-Leibniz formula as follows:

$$
\int_{t-\tau(t)}^{t} \dot{x}(s) d s=x(t)-x(t-\tau(t)) .
$$

By utilizing the zero equation, we obtain:

$$
\begin{gathered}
d_{1} \int_{t-\tau(t)}^{t} \dot{x}(s) d s=d_{1} x(t)-d_{1} x(t-\tau(t)), \\
\left(1-d_{2}\right) \int_{t-\tau(t)}^{t} \dot{x}(s) d s=\left(1-d_{2}\right) x(t)-\left(1-d_{2}\right) x(t-\tau(t)),
\end{gathered}
$$

where $d_{1}, d_{2} \in R$ will be selected to guarantee the exponential stability of Equation (4). By (9)-(11), Equation (4) can be formulated as follows:

$$
\begin{aligned}
\dot{x}(t)= & \left(d_{1}-a\right) x(t)+b \tanh x(t-\sigma(t))-\left(p+\dot{\tau}(t)-d_{2} \dot{\tau}(t)\right) \dot{x}(t-\tau(t)) \\
& -d_{1} x(t-\tau(t))-d_{1} \int_{t-\tau(t)}^{t} \dot{x}(s) d s+e \int_{t-h(t)}^{t} x(s) d s \\
& +f \int_{t-l(t)}^{t} \tanh x(s) d s .
\end{aligned}
$$


The following theorem presents the exponential stability for the neutral differential equation with time-varying delays in Equation (4).

Theorem 1. Consider $d_{1}, d_{2} \in R$ and positive real constants $\sigma_{1}, \sigma_{2}, \sigma_{d}, \tau_{1}, \tau_{2}, \tau_{d}, h_{1}, h_{2}, l_{1}, l_{2}$. Equation (4) is exponentially stable with a delay rate $\alpha>0$. For any positive real constants $\beta_{i}, \eta_{i}, i=1,2, \ldots, 10$ and real constants $w_{k}, y_{k}, k=1,2, \ldots, 10$ such that:

$$
\Xi<0,
$$

where $\Xi=\left[\Phi_{(i, j)}\right]$ and $\Phi_{(j, i)}=\Phi_{(i, j)}$,

$$
\begin{aligned}
& \Phi_{(1,1)}=2 \alpha \beta_{1}+2 \beta_{1} d_{1}-2 \beta_{1} a+2 w_{1} d_{1}-2 w_{1} a+\beta_{2}+\beta_{3}+\eta_{1}+\eta_{2}+\beta_{6} \tau_{2}^{2}+\beta_{7} \sigma_{2}^{2} \\
& \quad+\eta_{3} \tau_{1}^{2}+\eta_{4} \sigma_{1}^{2}-\beta_{8}+\beta_{9}+\beta_{10} \sigma_{2}^{2}+\eta_{6} \sigma_{1}^{2}+2 y_{2}+\eta_{7} h_{2}^{2}+\eta_{8} l_{2}^{2}+\eta_{9} h_{1}^{2}+\eta_{10} l_{1}^{2} \\
& \Phi_{(1,2)}=-w_{1}+w_{2} d_{1}-w_{2} a+y_{1} \\
& \Phi_{(1,3)}=\beta_{1} b+w_{1} b+w_{3} d_{1}-w_{3} a+y_{5} \\
& \Phi_{(1,4)}=-\beta_{1} d_{1}-w_{1} d_{1}+\beta_{8}-s-y_{2}+y_{3} \\
& \Phi_{(1,5)}=-\beta_{1} p-\beta_{1} \tau_{d}+\beta_{1} d_{2} \tau_{d}-w_{1} p-w_{1} \tau_{d}+w_{1} d_{2} \tau_{d}+w_{4} d_{1}-w_{4} a \\
& \Phi_{(1,6)}=-\beta_{1} d_{1}-w_{1} d_{1}+w_{5} d_{1}-w_{5} a-y_{2}+y_{4} \\
& \Phi_{(1,7)}=\beta_{1} e+w_{1} e \\
& \Phi_{(1,8)}=\beta_{1} f+w_{1} f \\
& \Phi_{(1,9)}=s \\
& \Phi_{(1,19)}=y_{6} \\
& \Phi_{(1,20)}=y_{7} \\
& \Phi_{(2,2)}=-2 w_{2}+\beta_{4}+\beta_{5}+\beta_{8} \tau_{2}^{2}+\eta_{5} \tau_{1}^{2} \\
& \Phi_{(2,3)}=w_{2} b-w_{3} \\
& \Phi_{(2,4)}=-w_{2} d_{1}-y_{1}, \\
& \Phi_{(2,5)}=-w_{2} p-w_{2} \tau_{d}+w_{2} d_{2} \tau_{d}-w_{4} \\
& \Phi_{(2,6)}=-w_{2} d_{1}-w_{5}-y_{1} \\
& \Phi_{(2,7)}=w_{2} e \\
& \Phi_{(2,8)}=w_{3} f \\
& \Phi_{(3,3)}=2 w_{3} b-\beta_{9} e^{-2 \alpha \sigma_{2}}+\beta_{9} \sigma_{d} \\
& \Phi_{(3,4)}=-w_{3} d_{1}-y_{5} \\
& \Phi_{(3,5)}=-w_{3} p-w_{3} \tau_{d}+w_{3} d_{2} \tau_{d}+w_{4} b \\
& \Phi_{(3,6)}=-w_{3} d_{1}+w_{5} b-y_{5} \\
& \Phi_{(3,7)}=w_{3} e \\
& \Phi_{(3,8)}=w_{3} f \\
& \Phi_{(4,4)}=-2 \beta_{8}+2 s-2 y_{3} \\
& \Phi_{(4,5)}=-w_{4} d_{1} \\
& \Phi_{(4,6)}=-w_{5} d_{1}-y_{3}-y_{4} \\
& \Phi_{(4,9)}=\beta_{8}-s \\
& \Phi_{(4,19)}=-y_{6} \\
& \Phi_{(4,20)}=-y_{7} \\
& \Phi_{(5,5)}=-2 w_{4} p-2 w_{4} \tau_{d}+2 w_{4} d_{2} \tau_{d}-\beta_{4} e^{-2 \alpha \tau_{2}}+\beta_{4} \tau_{d} \\
& \Phi_{(5,6)}=-w_{4} d_{1}-w_{5} p-w_{5} \tau_{d}-w_{5} d_{2} \tau_{d} \\
& \Phi_{(5,7)}=w_{4} e \\
& \Phi_{(5,8)}=w_{4} f \\
& \Phi_{(6,6)}=-2 w_{5} d_{1}-2 y_{4} \\
& \Phi_{(6,7)}=w_{5} e \\
& \Phi_{(6,8)}=w_{5} f \\
& \Phi_{(6,19)}=-y_{6} \\
& \Phi_{(6,20)}=-y_{7} \\
&
\end{aligned}
$$


$\Phi_{(8,8)}=-\eta_{8} e^{-2 \alpha l_{2}}$,

$\Phi_{(9,9)}=-\beta_{2} e^{-2 \alpha \tau_{2}}-\beta_{8}$,

$\Phi_{(10,10)}=-\beta_{3} e-2 \alpha \sigma_{2}$,

$\Phi_{(11,11)}=-\eta_{1} e^{-2 \alpha \tau_{1}}$,

$\Phi_{(12,12)}=-\eta_{2} e^{-2 \alpha \sigma_{1}}$,

$\Phi_{(13,13)}=-\beta_{5} e^{-2 \alpha \sigma_{2}}+\beta_{5} \sigma_{d}$,

$\Phi_{(14,14)}=-\beta_{6} e^{-2 \alpha \tau_{2}}$,

$\Phi_{(15,15)}=-\beta_{7} e^{-2 \alpha \sigma_{2}}$,

$\Phi_{(16,16)}=-\eta_{3} e^{-2 \alpha \tau_{1}}$,

$\Phi_{(17,17)}=-\eta_{4} e^{-2 \alpha \sigma_{1}}$,

$\Phi_{(18,18)}=-\eta_{5} e^{-2 \alpha \tau_{1}}$,

$\Phi_{(19,19)}=-\beta_{10} e^{-2 \alpha \sigma_{2}}$,

$\Phi_{(20,20)}=-\eta_{6} \sigma_{1} e^{-2 \alpha \sigma_{1}}$,

$\Phi_{(21,21)}=-\eta_{7} e^{-2 \alpha h_{2}}$,

$\Phi_{(22,22)}=-\eta_{8} e^{-2 \alpha l_{2}}$,

$\Phi_{(23,23)}=-\eta_{9} e^{-2 \alpha h_{1}}$,

$\Phi_{(24,24)}=-\eta_{10} e^{-2 \alpha l_{1}}$,

other terms are zero.

Proof. We study the following Lyapunov-Krasovskii functional for System (12):

$$
V\left(t, x_{t}\right)=\sum_{i=1}^{7} V_{i}\left(t, x_{t}\right)
$$

where:

$$
\begin{aligned}
V_{1}\left(t, x_{t}\right)= & \beta_{1} e^{2 \alpha t} x^{2}(t), \\
V_{2}\left(t, x_{t}\right)= & \beta_{2} \int_{t-\tau_{2}}^{t} e^{2 \alpha s} x^{2}(s) d s+\beta_{3} \int_{t-\sigma_{2}}^{t} e^{2 \alpha s} x^{2}(s) d s+\beta_{4} \int_{t-\tau(t)}^{t} e^{2 \alpha s} \dot{x}^{2}(s) d s \\
& +\beta_{5} \int_{t-\sigma(t)}^{t} e^{2 \alpha s} \dot{x}^{2}(s) d s+\eta_{1} \int_{t-\tau_{1}}^{t} e^{2 \alpha s} x^{2}(s) d s+\eta_{2} \int_{t-\sigma_{1}}^{t} e^{2 \alpha s} x^{2}(s) d s, \\
V_{3}\left(t, x_{t}\right)= & \beta_{6} \tau_{2} \int_{-\tau_{2}}^{0} \int_{t+\theta}^{t} e^{2 \alpha s} x^{2}(s) d s d \theta+\beta_{7} \sigma_{2} \int_{-\sigma_{2}}^{0} \int_{t+\theta}^{t} e^{2 \alpha s} x^{2}(s) d s d \theta \\
& +\eta_{3} \tau_{1} \int_{-\tau_{1}}^{0} \int_{t+\theta}^{t} e^{2 \alpha s} x^{2}(s) d s d \theta+\eta_{4} \sigma_{1} \int_{-\sigma_{1}}^{0} \int_{t+\theta}^{t} e^{2 \alpha s} x^{2}(s) d s d \theta, \\
V_{4}\left(t, x_{t}\right)= & \beta_{8} \tau_{2} \int_{-\tau_{2}}^{0} \int_{t+\theta}^{t} e^{2 \alpha s} \dot{x}^{2}(s) d s d \theta+\eta_{5} \tau_{1} \int_{-\tau_{1}}^{0} \int_{t+\theta}^{t} e^{2 \alpha s} \dot{x}^{2}(s) d s d \theta, \\
V_{5}\left(t, x_{t}\right)= & \beta_{9} \int_{t-\sigma(t)}^{t} e^{2 \alpha s} \tanh ^{2} x(s) d s+\beta_{10} \sigma_{2} \int_{-\sigma_{2}}^{0} \int_{t+\theta}^{t} e^{2 \alpha s} \tanh ^{2} x(s) d s d \theta \\
& +\eta_{6} \sigma_{1} \int_{-\sigma_{1}}^{0} \int_{t+\theta}^{t} e^{2 \alpha s} \tanh ^{2} x(s) d s d \theta, \\
& +\eta_{9} h_{1} \int_{-h_{1}}^{0} \int_{t+\theta}^{t} e^{2 \alpha s} x^{2}(s) d s d \theta+\eta_{10} l_{1} \int_{-l_{1}}^{0} \int_{t+\theta}^{t} e^{2 \alpha s} \tanh ^{2} x(s) d s d \theta, \\
V_{6}\left(t, x_{t}\right)= & \eta_{7} h_{2} \int_{-h_{2}}^{0} \int_{t+\theta}^{t} e^{2 \alpha s} x^{2}(s) d s d \theta+\eta_{8} l_{2} \int_{-l_{2}}^{0} \int_{t+\theta}^{t} e^{2 \alpha s} \tanh ^{2} x(s) d s d \theta \\
V_{7}\left(t, x_{t}\right)= & \gamma e^{2 \alpha t} x^{2}(t),
\end{aligned}
$$


where $\gamma$ is a positive number that will be found later. Taking the derivative of $V\left(t, x_{t}\right)$ along any trajectory of the solution of System (12), we get:

$$
\dot{V}\left(t, x_{t}\right)=\sum_{i=1}^{7} \dot{V}_{i}\left(t, x_{t}\right),
$$

where:

$$
\begin{aligned}
\dot{V}_{1}= & 2 \alpha \beta_{1} e^{2 \alpha t} x^{2}(t)+2 \beta_{1} e^{2 \alpha t} x(t) \dot{x}(t) \\
= & 2 \alpha \beta_{1} e^{2 \alpha t} x^{2}(t)+2 \beta_{1} e^{2 \alpha t} x(t)\left[d_{1} x(t)-a x(t)-d_{1} x(t-\tau(t))-d_{1} \int_{t-\tau(t)}^{t} \dot{x}(s) d s\right. \\
& +b \tanh x(t-\sigma(t))-p \dot{x}(t-\tau(t))+d_{2} \dot{\tau}(t) \dot{x}(t-\tau(t))-\dot{\tau}(t) \dot{x}(t-\tau(t)) \\
& \left.+e \int_{t-h(t)}^{t} x(s) d s+f \int_{t-l(t)}^{t} \tanh x(s) d s\right] \\
& +2 w_{1} e^{2 \alpha t} x(t)\left[d_{1} x(t)-a x(t)-\dot{x}(t)-d_{1} x(t-\tau(t))-d_{1} \int_{t-\tau(t)}^{t} \dot{x}(s) d s\right. \\
& +b \tanh x(t-\sigma(t))-p \dot{x}(t-\tau(t))+d_{2} \dot{\tau}(t) \dot{x}(t-\tau(t))-\dot{\tau}(t) \dot{x}(t-\tau(t)) \\
& \left.+e \int_{t-h(t)}^{t} x(s) d s+f \int_{t-l(t)}^{t} \tanh x(s) d s\right] \\
& +2 w_{2} e^{2 \alpha t} \dot{x}(t)\left[d_{1} x(t)-a x(t)-\dot{x}(t)-d_{1} x(t-\tau(t))-d_{1} \int_{t-\tau(t)}^{t} \dot{x}(s) d s\right. \\
& +b \tanh x(t-\sigma(t))-p \dot{x}(t-\tau(t))+d_{2} \dot{\tau}(t) \dot{x}(t-\tau(t))-\dot{\tau}(t) \dot{x}(t-\tau(t)) \\
& \left.+e \int_{t-h(t)}^{t} x(s) d s+f \int_{t-l(t)}^{t} \tanh x(s) d s\right] \\
& +2 w_{3} e^{2 \alpha t} \tanh x(t-\sigma(t))\left[d_{1} x(t)-a x(t)-\dot{x}(t)-d_{1} x(t-\tau(t))-d_{1} \int_{t-\tau(t)}^{t} \dot{x}(s) d s\right. \\
& +b \tanh x(t-\sigma(t))-p \dot{x}(t-\tau(t))+d_{2} \dot{\tau}(t) \dot{x}(t-\tau(t))-\dot{\tau}(t) \dot{x}(t-\tau(t)) \\
& \left.+e \int_{t-h(t)}^{t} x(s) d s+f \int_{t-l(t)}^{t} \tanh x(s) d s\right] \\
\dot{V}_{2}= & \beta_{2}\left[e^{2 \alpha t} x^{2}(t)-e^{2 \alpha\left(t-\tau_{2}\right)} x^{2}\left(t-\tau_{2}\right)\right]+\beta_{3}\left[e^{2 \alpha t} x^{2}(t)-e^{2 \alpha\left(t-\sigma_{2}\right)} x^{2}\left(t-\sigma_{2}\right)\right] \\
& +\beta_{4}\left[e^{2 \alpha t} \dot{x} \dot{x}^{2}(t)-\left(e^{2 \alpha(t-\tau(t))} \dot{x}^{2}(t-\tau(t))\right)(1-\dot{\tau}(t))\right] \\
& +\beta_{5}\left[e^{2 \alpha t} \dot{x}{ }^{2}(t)-\left(e^{2 \alpha(t-\sigma(t))} \dot{x}^{2}(t-\sigma(t))\right)(1-\dot{\sigma}(t))\right] \\
& +\eta_{1}\left[e^{2 \alpha t} x^{2}(t)-e^{2 \alpha\left(t-\tau_{1}\right)} x^{2}\left(t-\tau_{1}\right)\right]+\eta_{2}\left[e^{2 \alpha t} x^{2}(t)-e^{2 \alpha\left(t-\sigma_{1}\right)} x^{2}\left(t-\sigma_{1}\right)\right] . \\
& +2 w_{4} e^{2 \alpha t} \dot{x}(t-\tau(t))\left[d_{1} x(t)-a x(t)-\dot{x}(t)-d_{1} x(t-\tau(t))-d_{1} \int_{t-\tau(t)}^{t} \dot{x}(s) d s\right. \\
& +b \tanh x(t-\sigma(t))-p \dot{x}(t-\tau(t))+d_{2} \dot{\tau}(t) \dot{x}(t-\tau(t))-\dot{\tau}(t) \dot{x}(t-\tau(t)) \\
& \left.+e \int_{t-h(t)}^{t} x(s) d s+f \int_{t-l(t)}^{t} \tanh x(s) d s\right] \\
& +2 w_{5} e^{2 \alpha t} \int_{t-\tau(t)}^{t} \dot{x}(s) d s\left[d_{1} x(t)-a x(t)-\dot{x}(t)-d_{1} x(t-\tau(t))-d_{1} \int_{t-\tau(t)}^{t} \dot{x}(s) d s\right. \\
& +b \tanh x(t-\sigma(t))-p \dot{x}(t-\tau(t))+d_{2} \dot{\tau}(t) \dot{x}(t-\tau(t))-\dot{\tau}(t) \dot{x}(t-\tau(t)) \\
& \left.+e \int_{t}^{t} x(s) d s+f \int_{t-l(t)}^{t} \tanh x(s) d s\right] . \\
& \\
&
\end{aligned}
$$


Since $\tau_{1} \leq \tau(t) \leq \tau_{2}, \sigma_{1} \leq \sigma(t) \leq \sigma_{2}, \dot{\tau}(t)<\tau_{d}$ and $\dot{\sigma}(t)<\sigma_{d}$, we have:

$$
\begin{aligned}
\dot{V}_{2} & \leq e^{2 \alpha t}\left[\beta_{2} x^{2}(t)-\beta_{2} e^{-2 \alpha \tau_{2}} x^{2}\left(t-\tau_{2}\right)+\beta_{3} x^{2}(t)-\beta_{3} e^{-2 \alpha \sigma_{2}} x^{2}\left(t-\sigma_{2}\right)+\beta_{4} \dot{x}^{2}(t)\right. \\
& -\beta_{4} e^{-2 \alpha \tau_{2}} x^{2}(t-\tau(t))+\tau_{d} \beta_{4} e^{-2 \alpha \tau_{1}} \dot{x}^{2}(t-\tau(t))+\beta_{5} \dot{x}^{2}(t)-\beta_{5} e^{-2 \alpha \sigma_{2}} \dot{x}^{2}(t-\sigma(t)) \\
& +\sigma_{d} \beta_{5} e^{-2 \alpha \sigma_{1}} \dot{x}^{2}(t-\sigma(t))+\eta_{1} x^{2}(t)-\eta_{1} e^{-2 \alpha \tau_{1}} x^{2}\left(t-\tau_{1}\right)+\eta_{2} x^{2}(t) \\
& \left.-\eta_{2} e^{-2 \alpha \sigma_{1}} x^{2}\left(t-\sigma_{1}\right)\right] . \\
\dot{V}_{3}= & \beta_{6} \tau_{2}\left[\int_{-\tau_{2}}^{0} e^{2 \alpha t} x^{2}(t) d \theta-\int_{-\tau_{2}}^{0} e^{2 \alpha(t+\theta)} x^{2}(t+\theta) d \theta\right] \\
& +\beta_{7} \sigma_{2}\left[\int_{-\sigma_{2}}^{0} e^{2 \alpha t} x^{2}(t) d \theta-\int_{-\sigma_{2}}^{0} e^{2 \alpha(t+\theta)} x^{2}(t+\theta) d \theta\right] \\
& +\eta_{3} \tau_{1}\left[\int_{-\tau_{1}}^{0} e^{2 \alpha t} x^{2}(t) d \theta-\int_{-\tau_{1}}^{0} e^{2 \alpha(t+\theta)} x^{2}(t+\theta) d \theta\right] \\
& +\eta_{4} \sigma_{1}\left[\int_{-\sigma_{1}}^{0} e^{2 \alpha t} x^{2}(t) d \theta-\int_{-\sigma_{1}}^{0} e^{2 \alpha(t+\theta)} x^{2}(t+\theta) d \theta\right] .
\end{aligned}
$$

By Lemma 1, we get:

$$
\begin{aligned}
\dot{V}_{3} \leq & e^{2 \alpha t}\left[\beta_{6} \tau_{2}^{2} x^{2}(t)+\beta_{7} \sigma_{2}^{2} x^{2}(t)+\eta_{3} \tau_{1}^{2} x^{2}(t)+\eta_{4} \sigma_{1}^{2} x^{2}(t)\right. \\
& -\beta_{6} \tau_{2} e^{-2 \alpha \tau_{2}}\left(\int_{t-\tau_{2}}^{t} x(s) d s\right)^{2}-\beta_{7} \sigma_{2} e^{-2 \alpha \sigma_{2}}\left(\int_{t-\sigma_{2}}^{t} x(s) d s\right)^{2}-\eta_{3} \tau_{1} e^{-2 \alpha \tau_{1}}\left(\int_{t-\tau_{1}}^{t} x(s) d s\right)^{2} \\
& \left.-\eta_{4} \sigma_{1} e^{-2 \alpha \sigma_{1}}\left(\int_{t-\sigma_{1}}^{t} x(s) d s\right)^{2}\right] . \\
\dot{V}_{4}= & \beta_{8} \tau_{2}\left[\int_{-\tau_{2}}^{0} e^{2 \alpha t} \dot{x}^{2}(t) d \theta-\int_{-\tau_{2}}^{0} e^{2 \alpha(t+\theta)} \dot{x}^{2}(t+\theta) d \theta\right] \\
& +\eta_{5} \tau_{1}\left[\int_{-\tau_{1}}^{0} e^{2 \alpha t} \dot{x}^{2}(t) d \theta-\int_{-\tau_{1}}^{0} e^{2 \alpha(t+\theta)} \dot{x}^{2}(t+\theta) d \theta\right] .
\end{aligned}
$$

By Lemma 4, we get:

$$
\begin{aligned}
\dot{V}_{4} \leq & e^{2 \alpha t}\left[\beta_{8} \tau_{2}^{2} e^{2 \alpha \tau_{2}} \dot{x}^{2}(t)+\eta_{5} \tau_{1}^{2} \dot{x}(t)+\eta_{5} e^{-2 \alpha \tau_{1}}\left(\int_{t-\tau_{1}}^{t} \dot{x}(s) d s\right)^{2}\right] \\
& +e^{2 \alpha t}\left[\begin{array}{lll}
x(t) & x(t-\tau(t)) & x\left(t-\tau_{2}\right)
\end{array}\right] \\
& {\left[\begin{array}{ccc}
-\beta_{8} & \beta_{8}-s & s \\
* & -2 \beta_{8}+2 s & \beta_{8}-s \\
* & * & -\beta_{8}
\end{array}\right]\left[\begin{array}{c}
x(t) \\
x(t-\tau(t)) \\
x\left(t-\tau_{2}\right)
\end{array}\right] . } \\
\dot{V}_{5}= & \beta_{9} e^{2 \alpha t} \tanh ^{2} x(t)-\beta_{9} e^{2 \alpha(t-\sigma(t)} \tanh ^{2} x(t-\sigma(t)) \\
& +\beta_{9} \dot{\sigma}(t) e^{2 \alpha(t-\sigma(t))} \tanh ^{2} x(t-\sigma(t))+\beta_{10} \sigma_{2} \int_{-\sigma_{2}}^{0} e^{2 \alpha t} \tanh ^{2} x(t) d \theta \\
& -\beta_{10} \sigma_{2} \int_{-\sigma_{2}}^{0} e^{2 \alpha(t+\theta)} \tanh ^{2} x(t+\theta) d \theta+\eta_{6} \sigma_{1} \int_{-\sigma_{1}}^{0} e^{2 \alpha t} \tanh ^{2} x(t) d \theta \\
& -\eta_{6} \sigma_{1} \int_{-\sigma_{1}}^{0} e^{2 \alpha(t+\theta)} \tanh ^{2} x(t+\theta) d \theta .
\end{aligned}
$$


Since $\tanh ^{2} x(t) \leq x^{2}(t)$ and using Lemma 1, we get:

$$
\begin{aligned}
\dot{V}_{5} \leq & e^{2 \alpha t}\left[\beta_{9} x^{2}(t)-\beta_{9} e^{-2 \alpha \sigma_{2}} \tanh ^{2} x(t-\sigma(t))+\beta_{9} \sigma_{d} e^{-2 \alpha \sigma_{1}} \tanh ^{2} x(t-\sigma(t))\right. \\
& +\beta_{10} \sigma_{2}^{2} x^{2}(t)-\beta_{10} \sigma_{2} e^{-2 \alpha \sigma_{2}}\left(\int_{t-\sigma_{2}}^{t} \tanh x(s) d s\right)^{2}+\eta_{6} \sigma_{1}^{2} x^{2}(t) \\
& \left.-\eta_{6} \sigma_{1} e^{-2 \alpha \sigma_{1}}\left(\int_{t-\sigma_{1}}^{t} \tanh x(s) d s\right)^{2}\right] . \\
\dot{V}_{6}= & \eta_{7} h_{2}\left[\int_{-h_{2}}^{0}\left(e^{2 \alpha t} x^{2}(t)-e^{2 \alpha(t+\theta)} x^{2}(t+\theta)\right) d \theta\right] \\
& +\eta_{8} l_{2}\left[\int_{-l_{2}}^{0}\left(e^{2 \alpha t} \tanh ^{2} x(t)-e^{2 \alpha(t+\theta)} \tanh ^{2} x(t+\theta)\right) d \theta\right] \\
& +\eta_{9} h_{1}\left[\int_{-h_{1}}^{0}\left(e^{2 \alpha t} x^{2}(t)-e^{2 \alpha(t+\theta)} x^{2}(t+\theta)\right) d \theta\right] \\
& +\eta_{10} l_{1}\left[\int_{-l_{1}}^{0}\left(e^{2 \alpha t} \tanh ^{2} x(t)-e^{2 \alpha(t+\theta)} \tanh ^{2} x(t+\theta)\right) d \theta\right] .
\end{aligned}
$$

Since $\tanh ^{2} x(t) \leq x^{2}(t)$ and using Lemma 3, we get:

$$
\begin{aligned}
\dot{V}_{6} \leq & e^{2 \alpha t}\left[\eta_{7} h_{2}^{2} x^{2}(t)-\eta_{7} e^{-2 \alpha h_{2}}\left(\int_{t-h(t)}^{t} x(s) d s\right)^{2}-\eta_{7} e^{-2 \alpha h_{2}}\left(\int_{t-h_{2}}^{t-h(t)} x(s) d s\right)^{2}\right] \\
& +e^{2 \alpha t}\left[\left[\eta_{8} l_{2}^{2} x^{2}(t)-\eta_{8} e^{-2 \alpha l_{2}}\left(\int_{t-l(t)}^{t} \tanh x(s) d s\right)^{2}\right.\right. \\
& \left.-\eta_{8} e^{-2 \alpha l_{2}}\left(\int_{t-l_{2}}^{t-l(t)} \tanh x(s) d s\right)^{2}\right]+e^{2 \alpha t}\left[\eta_{9} h_{1}^{2} x^{2}(t)\right. \\
& \left.-\eta_{9} e^{-2 \alpha h_{1}}\left(\int_{t-h_{1}}^{t} x(s) d s\right)^{2}\right]+e^{2 \alpha t}\left[\eta_{10} l_{1}^{2} x^{2}(t)-\eta_{10} e^{-2 \alpha l_{1}}\left(\int_{t-l_{1}}^{t} \tanh x(s) d s\right)^{2}\right] .
\end{aligned}
$$

From the Newton-Leibniz formula, we have:

$$
\begin{aligned}
& 2\left[y_{1} \dot{x}(t)+y_{2} x(t)+y_{3} x(t-\tau(t))+y_{4} \int_{t-\tau(t)}^{t} \dot{x}(s) d s+y_{5} \tanh x(t-\sigma(t))\right. \\
& \left.+y_{6} \int_{t-\sigma_{2}}^{t} \tanh x(s) d s+y_{7} \int_{t-\sigma_{1}}^{t} \tanh x(s) d s\right]\left[x(t)-x(t-\tau(t))-\int_{t-\tau(t)}^{t} \dot{x}(s) d s\right] .
\end{aligned}
$$

Combining Equations (16)-(22), we get:

$$
\dot{V}^{*}=\sum_{i=1}^{6} V_{i}\left(t, x_{t}\right) \leq e^{2 \alpha t} \omega^{T}(t) \Xi \omega(t),
$$

where $\omega(t)=\left[x(t), \dot{x}(t), \tanh x(t-\sigma(t)), x(t-\tau(t)), \dot{x}(t-\tau(t)), \int_{t-\tau(t)}^{t} \dot{x}(s) d s, \int_{t-h(t)}^{t} x(s) d s\right.$, $\int_{t-l(t)}^{t} \tanh x(s) d s, x\left(t-\tau_{2}\right), x\left(t-\sigma_{2}\right), x\left(t-\tau_{1}\right), x\left(t-\sigma_{1}\right), \dot{x}(t-\sigma(t)), \int_{t-\tau_{2}}^{t} x(s) d s, \int_{t-\sigma_{2}}^{t} x(s) d s$, $\int_{t-\tau_{1}}^{t} x(s) d s, \int_{t-\sigma_{1}}^{t} x(s) d s, \int_{t-\sigma_{1}}^{t} \dot{x}(s) d s, \int_{t-\sigma_{2}}^{t} \tanh x(s) d s, \int_{t-\sigma_{1}}^{t} \tanh x(s) d s, \int_{t-h_{2}}^{t-h(t)} x(s) d s$, $\left.\int_{t-l_{2}}^{t-l(t)} \tanh x(s) d s, \int_{t-h_{1}}^{t} x(s) d s, \int_{t-l_{1}}^{t} \tanh x(s) d s\right]^{T}$, 
and $\Xi$ is identified in Equation (13). Due to $\Xi<0$, we get $\dot{V}^{*} \leq e^{2 \alpha t} \omega^{T}(t) \Xi \omega(t)<0$. Hence, there is a positive number $\lambda$ such that:

$$
\begin{aligned}
\dot{V}^{*} \leq & -\lambda e^{2 \alpha t}\left[\|\dot{x}(t)\|^{2}+\|x(t)\|^{2}+\|\tanh x(t-\sigma(t))\|^{2}+\|x(t-\tau(t))\|^{2}+\|\dot{x}(t-\tau(t))\|^{2}\right. \\
& +\left\|\int_{t-\tau(t)}^{t} \dot{x}(s) d s\right\|^{2}+\left\|\int_{t-h(t)}^{t} x(s) d s\right\|^{2}+\|\dot{x}(t-\sigma(t))\|^{2}+\left\|\int_{t-\tau_{2}}^{t} x(s) d s\right\|^{2} \\
& +\left\|\int_{t-\sigma_{2}}^{t} x(s) d s\right\|^{2}+\left\|\int_{t-l(t)}^{t} \tanh x(s) d s\right\|^{2}+\left\|x\left(t-\tau_{2}\right)\right\|^{2}+\left\|x\left(t-\sigma_{2}\right)\right\|^{2} \\
& +\left\|x\left(t-\tau_{1}\right)\right\|^{2}+\left\|x\left(t-\sigma_{1}\right)\right\|^{2}+\left\|\int_{t-\tau_{1}}^{t} x(s) d s\right\|^{2}+\left\|\int_{t-\sigma_{1}}^{t} x(s) d s\right\|^{2} \\
& +\left\|\int_{t-\sigma_{1}}^{t} \dot{x}(s) d s\right\|^{2}+\left\|\int_{t-\sigma_{2}}^{t} \tanh x(s) d s\right\|^{2}+\left\|\int_{t-\sigma_{1}}^{t} \tanh x(s) d s\right\|^{2} \\
& +\left\|\int_{t-h(t)}^{t-h(t)} x(s) d s\right\|^{2}+\left\|\int_{t-l_{2}}^{t-l(t)} \tanh x(s) d s\right\|^{2}+\left\|\int_{t-h_{1}}^{t} x(s) d s\right\|^{2} \\
& \left.+\left\|\int_{t-l_{1}}^{t} \tanh x(s) d s\right\|^{2}\right] \\
& \leq-\lambda e^{2 \alpha t}\|x(t)\|^{2} .
\end{aligned}
$$

Calculating the derivative of $V_{7}$ along any trajectory of Equation (4) and applying Lemma 2, then we obtain:

$$
\begin{aligned}
\dot{V}_{7}= & 2 \gamma e^{2 \alpha t}\left[x(t) \dot{x}(t)+\alpha x^{2}(t)\right] \\
= & 2 \gamma e^{2 \alpha t}\left[x ( t ) \left(-a x(t)+b \tanh x(t-\sigma(t))-p \dot{x}(t-\tau(t))+e \int_{t-h(t)}^{t} x(s) d s\right.\right. \\
& \left.\left.+f \int_{t-l(t)}^{t} \tanh x(s) d s\right)+\alpha x^{2}(t)\right] \\
= & 2 \gamma e^{2 \alpha t}\left[-a x^{2}(t)+b x(t) \tanh x(t-\sigma(t))-p x(t) \dot{x}(t-\tau(t))+e x(t) \int_{t-h(t)}^{t} x(s) d s\right. \\
& \left.+f x(t) \int_{t-l(t)}^{t} \tanh x(s) d s+\alpha x^{2}(t)\right] \\
\leq & \gamma e^{2 \alpha t}\left[(-2 a+2 \alpha) x^{2}(t)+b^{2} \tanh ^{2} x(t-\sigma(t))+p^{2} \dot{x}^{2}(t-\tau(t))\right. \\
& \left.+e^{2}\left(\int_{t-h(t)}^{t} x(s) d s\right)^{2}+f^{2}\left(\int_{t-l(t)}^{t} \tanh x(s) d s\right)^{2}\right] .
\end{aligned}
$$

We select:

$$
\gamma=\left\{\begin{array}{l}
\frac{\lambda}{2} \min \left\{\frac{1}{b^{2}}, \frac{1}{p^{2}}, \frac{1}{e^{2}}, \frac{1}{f^{2}}\right\}, \quad \text { if }-2 a+2 \alpha \leq 0 \\
\frac{\lambda}{2} \min \left\{\frac{1}{-2 a+2 \alpha}, \frac{1}{b^{2}}, \frac{1}{p^{2}} \frac{1}{e^{2}}, \frac{1}{f^{2}}\right\}, \quad \text { if otherwise }
\end{array}\right.
$$


We have $\dot{V}\left(t, x_{t}\right)=\sum_{i=1}^{7} V_{i}\left(t, x_{t}\right) \leq-\frac{\lambda}{2} e^{2 \alpha t}\|x(t)\|^{2}<0$. From the conditions that $0 \leq \tau(t) \leq$ $\tau_{2}, 0 \leq \sigma(t) \leq \sigma_{2}$ and $\dot{V}\left(t, x_{t}\right)$ is negative definite, we get $V\left(t, x_{t}\right) \leq V\left(0, x_{0}\right), \forall t \geq 0$, with:

$$
\begin{aligned}
& V\left(0, x_{0}\right)=\sum_{i=1}^{7} V_{i}\left(0, x_{0}\right) \\
& =\beta_{1} x^{2}(0)+\beta_{2} \int_{-\tau_{2}}^{t} e^{2 \alpha s} x^{2}(s) d s+\beta_{3} \int_{-\sigma_{2}}^{0} e^{2 \alpha s} x^{2}(s) d s \\
& +\beta_{4} \int_{-\tau(0)}^{0} e^{2 \alpha s} \dot{x}^{2}(s) d s+\beta_{5} \int_{-\sigma(0)}^{t} e^{2 \alpha s} \dot{x}^{2}(s) d s \\
& +\eta_{1} \int_{-\tau(0)}^{0} e^{2 \alpha s} x^{2}(s) d s+\eta_{2} \int_{-\sigma(0)}^{t} e^{2 \alpha s} x^{2}(s) d s \\
& +\beta_{6} \tau_{2} \int_{-\tau_{2}}^{0} \int_{\theta}^{0} e^{2 \alpha s} x^{2}(s) d s d \theta+\beta_{7} \sigma_{2} \int_{-\sigma_{2}}^{0} \int_{\theta}^{0} e^{2 \alpha s} x^{2}(s) d s d \theta \\
& +\eta_{3} \tau_{1} \int_{-\tau_{1}}^{0} \int_{\theta}^{0} e^{2 \alpha s} x^{2}(s) d s d \theta+\eta_{4} \sigma_{1} \int_{-\sigma_{1}}^{0} \int_{\theta}^{0} e^{2 \alpha s} x^{2}(s) d s d \theta \\
& +\beta_{8} \tau_{2} \int_{-\tau_{2}}^{0} \int_{\theta}^{0} e^{2 \alpha s} \dot{x}^{2}(s) d s d \theta+\eta_{5} \tau_{1} \int_{-\tau_{1}}^{0} \int_{\theta}^{0} e^{2 \alpha s} \dot{x}^{2}(s) d s d \theta \\
& +\beta_{9} \int_{-\sigma(0)}^{0} e^{2 \alpha s} \tanh ^{2} x(s) d s+\beta_{10} \sigma_{2} \int_{-\sigma_{2}}^{0} \int_{\theta}^{0} e^{2 \alpha s} \tanh ^{2} x(s) d s d \theta \\
& +\eta_{6} \sigma_{1} \int_{-\sigma_{1}}^{0} \int_{\theta}^{0} e^{2 \alpha s} \tanh ^{2} x(s) d s d \theta+\eta_{7} h_{2} \int_{-h_{2}}^{0} \int_{\theta}^{0} e^{2 \alpha s} x^{2}(s) d s d \theta \\
& +\eta_{8} l_{2} \int_{-l_{2}}^{0} \int_{\theta}^{0} e^{2 \alpha s} \tanh ^{2} x(s) d s d \theta+\eta_{9} h_{1} \int_{-h_{1}}^{0} \int_{\theta}^{0} e^{2 \alpha s} x^{2}(s) d s d \theta \\
& +\eta_{10} l_{1} \int_{-l_{1}}^{0} \int_{\theta}^{0} e^{2 \alpha s} \tanh ^{2} x(s) d s d \theta+\gamma x^{2}(0) \\
& \leq\left[\beta_{1}+\beta_{2} \tau_{2}+\beta_{3} \sigma_{2}+\beta_{4} \tau_{2}+\beta_{5} \sigma_{2}+\eta_{1} \tau_{1}+\eta_{1} \sigma_{1}+\beta_{6} \frac{\tau_{2}^{3}}{2}+\beta_{7} \frac{\sigma_{2}^{3}}{2}+\eta_{3} \frac{\tau_{1}^{3}}{2}\right. \\
& +\eta_{4} \frac{\sigma_{1}^{3}}{2}+\beta_{8} \frac{\tau_{2}^{3}}{2}+\eta_{5} \frac{\sigma_{1}^{3}}{2}+\beta_{9} \sigma_{2}+\beta_{10} \frac{\sigma_{2}^{3}}{2}+\eta_{6} \frac{\sigma_{1}^{3}}{2}+\eta_{7} \frac{h_{2}^{3}}{2}+\eta_{8} \frac{l_{2}^{3}}{2}+\eta_{9} \frac{h_{1}^{3}}{2} \\
& \left.+\eta_{10} \frac{l_{1}^{3}}{2}+\gamma\right] \max \left\{\|x\|_{s}^{2},\|\dot{x}\|_{l}^{2}\right\} \\
& =\Delta \max \left\{\|x\|_{s}^{2},\|\dot{x}\|_{l}^{2}\right\} \\
& =\Delta N \text {, }
\end{aligned}
$$

where $\Delta=\beta_{1}+\beta_{2} \tau_{2}+\beta_{3} \sigma_{2}+\beta_{4} \tau_{2}+\beta_{5} \sigma_{2}+\eta_{1} \tau_{1}+\eta_{1} \sigma_{1}+\beta_{6} \frac{\tau_{2}^{3}}{2}+\beta_{7} \frac{\sigma_{2}^{3}}{2}+\eta_{3} \frac{\tau_{1}^{3}}{2}+\eta_{4} \frac{\sigma_{1}^{3}}{2}+\beta_{8} \frac{\tau_{2}^{3}}{2}+$ $\eta_{5} \frac{\sigma_{1}^{3}}{2}+\beta_{9} \sigma_{2}+\beta_{10} \frac{\sigma_{2}^{3}}{2}+\eta_{6} \frac{\sigma_{1}^{3}}{2}+\eta_{7} \frac{h_{2}^{3}}{2}+\eta_{8} \frac{l_{2}^{3}}{2}+\eta_{9} \frac{h_{1}^{3}}{2}+\eta_{10} \frac{l_{1}^{3}}{2}+\gamma, N=\max \left\{\|x\|_{s}^{2},\|\dot{x}\|_{l}^{2}\right\}$.

From $\gamma e^{2 \alpha t} x^{2}(t) \leq V(x(t)) \leq \Delta N$, we obtain:

$$
\|x(t)\| \leq M e^{-\alpha t}, \quad M=\sqrt{\frac{\Delta N}{\gamma}} .
$$

This completes the poof.

We show the delay-dependent criteria for the problem of the exponential stability of Equation (4) where $\tau_{1}=\sigma_{1}=e=f=0$. 
Corollary 1. Consider real constants $d_{1} \in R$ and positive real constants $\tau_{2}, \tau_{d}, \sigma_{2}$ and $\sigma_{d}$. Equation (4) is exponentially stable with a delay rate $\alpha>0$, for any positive real constants $\beta_{i}, i=1,2, \ldots, 10$ and real constants $w_{k}, m_{k}, k=1,2, \ldots, 6$ such that:

$$
\Xi^{*}<0
$$

where $\Xi^{*}=\left[\Phi_{(i, j)}^{*}\right]$ and $\left[\Phi_{(j, i)}^{*}\right]=\left[\Phi_{(i, j)}^{*}\right]$,

$\Phi_{(1,1)}^{*}=-2 w_{2}+\beta_{4}+\beta_{5}+\beta_{8} \tau_{2} \tau_{2}-2 w_{6}$,

$\Phi_{(1,2)}^{*}=-w_{1}+w_{2} d_{1}-w_{2} a+y_{1}-w_{6} a$,

$\Phi_{(1,3)}^{*}=-w_{2} d_{1}-y_{1}$,

$\Phi_{(1,5)}^{*}=-w_{2} d_{1}-w_{5}-y_{1}$,

$\Phi_{(1,6)}^{*}=w_{2} b-w_{3}+w_{6} b$,

$\Phi_{(1,7)}^{*}=-w_{2} p-w_{4}-w_{6} p$,

$\Phi_{(2,2)}^{*}=2 \alpha \beta_{1}+2 \beta_{1} d_{1}-2 \beta_{1} a+2 w_{1} d_{1}-2 w_{1} a+\beta_{2}+\beta_{3}+\beta_{6} \tau_{2} \tau_{2}+\beta_{7} \sigma_{2} \sigma_{2}-\beta_{8} e^{-2 \alpha \tau_{2}}+\beta_{9}$

$+\beta_{10} \sigma_{2} \sigma_{2}+2 y_{2}$

$\Phi_{(2,3)}^{*}=-\beta_{1} d_{1}-w_{1} d_{1}+\beta_{8} e^{-2 \alpha \tau_{2}}-s_{1}-y_{2}+y_{3}$

$\Phi_{(2,4)}^{*}=s_{1}$,

$\Phi_{(2,5)}^{*}=-\beta_{1} d_{1}-w_{1} d_{1}+w_{5} d_{1}-a w_{5}-y_{2}+y_{4}$

$\Phi_{(2,6)}^{*}=\beta_{1} b+w_{1} b+w_{3} d_{1}-w_{3} a+y_{5}$

$\Phi_{(2,7)}^{*}=-\beta_{1} p-w_{1} p+w_{4} d_{1}-w_{4} a$,

$\Phi_{(2,12)}^{*}=y_{6}$

$\Phi_{(3,3)}^{*}=-2 \beta_{8} e^{-2 \alpha \tau_{2}}+2 s_{1}-2 y_{3}$,

$\Phi_{(3,4)}^{*}=\beta_{8} e^{-2 \alpha \tau_{2}}-s_{1}$,

$\Phi_{(3,5)}^{*}=-w_{5} d_{1}-y_{3}-y_{4}$

$\Phi_{(3,6)}^{*}=-w_{3} d_{1}-y_{5}$,

$\Phi_{(3,7)}^{*}=-w_{4} d_{1}$,

$\Phi_{(3,12)}^{*}=-y_{6}$

$\Phi_{(4,4)}^{*}=-\beta_{2} e^{-2 \alpha \tau_{2}}-\beta_{8} e^{-2 \alpha \tau_{2}}$,

$\Phi_{(5,5)}^{*}=-2 w_{5} d_{1}-2 y_{4}$

$\Phi_{(5,6)}^{*}=-w_{3} d_{1}+w_{5} b-y_{5}$,

$\Phi_{(5,7)}^{*}=-w_{4} d_{1}-w_{5} p$,

$\Phi_{(5,12)}^{*}=-y_{6}$,

$\Phi_{(6,6)}^{*}=2 w_{3} b-\beta_{9} e^{-2 \alpha \sigma_{2}}+\beta_{9} \sigma_{d}$,

$\Phi_{(6,7)}^{*}=-w_{3} p+w_{4} b$,

$\Phi_{(7,7)}^{*}=-2 w_{4} p+\tau_{d} \beta_{4}-\beta_{4} e^{-2 \alpha \tau_{2}}$,

$\Phi_{(8,8)}^{*}=-\beta_{3} e^{-2 \alpha \sigma_{2}}$

$\Phi_{(9,9)}^{*}=-\beta_{5} e^{-2 \alpha \sigma_{2}}+\sigma_{d} \beta_{5}$,

$\Phi_{(10,10)}^{*}=-\beta_{6} e^{-2 \alpha \tau_{2}}$,

$\Phi_{(11,11)}^{*}=-\beta_{7} e^{-2 \alpha \sigma_{2}}$,

$\Phi_{(12,12)}^{*}=-\beta_{10} e^{-2 \alpha \sigma_{2}}$

other terms are zero. 
Proof. Consider the Lyapunov-Krasovskii functional candidates:

$$
V\left(t, x_{t}\right)=\sum_{i=1}^{6} V_{i}\left(t, x_{t}\right)
$$

where:

$$
\begin{aligned}
V_{1}\left(t, x_{t}\right)= & \beta_{1} e^{2 \alpha t} x^{2}(t) \\
V_{2}\left(t, x_{t}\right)= & \beta_{2} \int_{t-\tau_{2}}^{t} e^{2 \alpha s} x^{2}(s) d s+\beta_{3} \int_{t-\sigma_{2}}^{t} e^{2 \alpha s} x^{2}(s) d s+\beta_{4} \int_{t-\tau(t)}^{t} e^{2 \alpha s} \dot{x}^{2}(s) d s \\
& +\beta_{5} \int_{t-\sigma(t)}^{t} e^{2 \alpha s} \dot{x}^{2}(s) d s, \\
V_{3}\left(t, x_{t}\right)= & \beta_{6} \tau_{2} \int_{-\tau_{2}}^{0} \int_{t+\theta}^{t} e^{2 \alpha(s-\theta)} x^{2}(s) d s d \theta+\beta_{7} \sigma_{2} \int_{-\sigma_{2}}^{o} \int_{t+\theta}^{t} e^{2 \alpha(s-\theta)} x^{2}(s) d s d \theta, \\
V_{4}\left(t, x_{t}\right)= & \beta_{8} \tau_{2} \int_{-\tau_{2}}^{0} \int_{t+\theta}^{t} e^{2 \alpha(s-\theta)} \dot{x}^{2}(s) d s d \theta, \\
V_{5}\left(t, x_{t}\right)= & \beta_{9} \int_{t-\sigma t}^{t} e^{2 \alpha s} \tanh ^{2} x(s) d s+\beta_{10} \sigma_{2} \int_{-\sigma_{2}}^{0} \int_{t+\theta}^{t} e^{2 \alpha(s-\theta)} \tanh ^{2} x(s) d s d \theta, \\
V_{6}\left(t, x_{t}\right)= & \gamma e^{2 \alpha t} x^{2}(t) .
\end{aligned}
$$

As stated by Theorem 1, we get the delay-dependent criteria for the exponential stability of Equation (4) where $\tau_{1}=\sigma_{1}=e=f=0$.

Remark 2. We know that the delay-dependent condition is generally considered as less conservative than the delay-independent when the delay is small. Therefore, our criteria in Theorem 1 and Corollary 1 are delay-dependent on $\tau_{2}, \sigma_{2}, h_{2}$, and $l_{2}$, which are less conservative than the delay-independent criteria considered in $[2,6,8,27]$.

Remark 3. We know that unbounded delays are more general than bounded delays, often including bounded delays as their special cases. In practical systems, there are many works such as the HIV-spread model, the modeling of oscillators, and the analysis of neural networks where infinite delays exist or modeling of one is need. Therefore, it is worth pointing out that we can extend this method to a more general system with infinite delays in future works.

\section{Numerical Examples}

In this part, numerical examples are presented to show the effectiveness of our main consequences, which compare the maximum allowable upper bounds $\sigma$ and the parameter $b$ in addition to investigating the rate of convergence. The feasibility of all criteria is obtained by using the LMI control toolbox in MATLAB.

Example 1. Consider the neutral differential equation with mixed interval time-varying delays as follows:

$$
\frac{d}{d t}[x(t)+0.2 x(t-\tau(t))]+0.6 x(t)-b \tanh x(t-\sigma(t))-0.1 \int_{t-h(t)}^{t} x(s) d s-0.2 \int_{t-l(t)}^{t} \tanh x(s) d s=0 .
$$

Solving the LMI (13) when $b=0.15, \alpha=0.0038, \tau(t)=0.1+\frac{\cos ^{2}(t)}{5}, \sigma(t)=0.1+\frac{\sin ^{2}(t)}{5}$, $h(t)=0.1+\frac{|\cos (2 t)|}{3}$ and $l(t)=0.1+\frac{|\sin (2 t)|}{3}$, we get a set of parameters to guarantee the exponential 
stability as follows:

$$
\begin{array}{llll}
\eta_{1}=108.2939, & \eta_{2}=108.2939, & \eta_{3}=264.6443, & \eta_{4}=264.6443, \\
\eta_{5}=268.6166, & \eta_{6}=505.1453, & \eta_{7}=290.0963, & \eta_{8}=427.4140, \\
\eta_{9}=264.6443, & \eta_{10}=264.6443, & \beta_{1}=1.5575 \times 10^{3}, & \beta_{2}=104.3766, \\
\beta_{3}=108.3823, & \beta_{4}=244.4721, & \beta_{5}=338.3978, & \beta_{6}=173.6726, \\
\beta_{7}=173.6726, & \beta_{8}=248.9316, & \beta_{9}=336.7202, & \beta_{10}=173.6726, \\
w_{1}=-229.6065, & w_{2}=487.9487, & w_{3}=124.9935, & w_{4}=-1.5458 \times 10^{-10}, \\
w_{5}=-14.5680, & &
\end{array}
$$

Moreover, Table 1 lists the maximum upper bounds $b$ for different values of $\sigma, \tau_{d}, \sigma_{d}$. Table 2 lists the maximum upper bounds for the rate of convergent $\sigma$ with different values of $b, \tau_{d}, \sigma_{d}$. The maximum allowable bounds on $b$ for different values of $\alpha, \tau_{d}, \sigma_{d}$ can be found in Table 3 . We investigate the maximum allowable bounds on the rate of convergent $\alpha$ in Table 4 with different values of $b, \tau_{d}, \sigma_{d}$. In Table 5 , we show the maximum allowable bounds on $b$ for the exponential and asymptotic stabilities of Example 1 for different values of $\alpha, e$. Table 6 provides the maximum allowable bounds on $\alpha$ for the exponential and asymptotic stabilities of Example 1 for different values of $e, b$. Table 7 provides the maximum allowable bounds on $b$ for the asymptotic and exponential stabilities of Example 1 for different values of $\alpha, f$. Furthermore, Table 8 presents the maximum allowable bounds on $\alpha$ for the asymptotic and exponential stabilities of Example 1 for different values of $f, b$.

Table 1. Maximum allowable upper bounds $b$ of Example 1 for $\alpha=0.0038$.

\begin{tabular}{cccccc}
\hline $\boldsymbol{\tau}_{\boldsymbol{d}}=\sigma_{\boldsymbol{d}}$ & $\sigma=\mathbf{0 . 2}$ & $\sigma=\mathbf{0 . 3}$ & $\sigma=\mathbf{0 . 4}$ & $\sigma=\mathbf{0 . 5}$ & $\sigma=\mathbf{0 . 6}$ \\
\hline 0.1 & 0.4226 & 0.4224 & 0.4223 & 0.4221 & 0.4220 \\
0.2 & 0.3984 & 0.3990 & 0.3978 & 0.3979 & 0.3970 \\
0.3 & 0.3726 & 0.3724 & 0.3722 & 0.3720 & 0.3719 \\
0.4 & 0.3460 & 0.3447 & 0.3445 & 0.3443 & 0.3440 \\
0.5 & 0.3148 & 0.3145 & 0.3141 & 0.3142 & 0.3138 \\
\hline
\end{tabular}

Table 2. Maximum allowable upper bounds $\sigma$ of Example 1 for $\alpha=0.0038$.

\begin{tabular}{ccccc}
\hline $\boldsymbol{\tau}_{\boldsymbol{d}}=\sigma_{\boldsymbol{d}}$ & $\boldsymbol{b}=\mathbf{0 . 1}$ & $\boldsymbol{b}=\mathbf{0 . 2}$ & $\boldsymbol{b}=\mathbf{0 . 3}$ & $\boldsymbol{b}=\mathbf{0 . 4}$ \\
\hline 0.05 & 302.5884 & 181.7944 & 90.5145 & 20.6635 \\
0.1 & 249.3654 & 157.9067 & 78.0361 & 13.1826 \\
0.15 & 211.5763 & 137.6965 & 66.6394 & 6.1042 \\
0.2 & 182.2537 & 120.1813 & 56.1530 & 4.0409 \\
\hline
\end{tabular}

Table 3. Maximum allowable upper bounds $b$ of Example 1.

\begin{tabular}{cccccc}
\hline $\boldsymbol{\tau}_{\boldsymbol{d}}=\sigma_{\boldsymbol{d}}$ & $\boldsymbol{\alpha}=\mathbf{0}$ & $\boldsymbol{\alpha}=\mathbf{0 . 1}$ & $\boldsymbol{\alpha}=\mathbf{0 . 2}$ & $\boldsymbol{\alpha}=\mathbf{0 . 3}$ & $\boldsymbol{\alpha}=\mathbf{0 . 4}$ \\
\hline 0.1 & 0.4269 & 0.3036 & 0.1950 & 0.0971 & 0.0130 \\
0.2 & 0.4024 & 0.2838 & 0.1793 & 0.0886 & 0.0108 \\
0.3 & 0.3764 & 0.2622 & 0.1633 & 0.0792 & 0.0094 \\
0.4 & 0.3485 & 0.2410 & 0.1456 & 0.0685 & 0.0078 \\
0.5 & 0.3181 & 0.2129 & 0.1253 & 0.0558 & 0.0057 \\
\hline
\end{tabular}

Table 4. Maximum allowable upper bounds $\alpha$ of Example 1.

\begin{tabular}{cccccc}
\hline $\boldsymbol{\tau}_{\boldsymbol{d}}=\sigma_{\boldsymbol{d}}$ & $\boldsymbol{b}=\mathbf{0 . 0 5}$ & $\boldsymbol{b}=\mathbf{0 . 1}$ & $\boldsymbol{b}=\mathbf{0 . 2}$ & $\boldsymbol{b}=\mathbf{0 . 3}$ & $\boldsymbol{b}=\mathbf{0 . 4}$ \\
\hline 0.05 & 0.3563 & 0.3012 & 0.2009 & 0.1111 & 0.0296 \\
0.1 & 0.3538 & 0.2969 & 0.1942 & 0.1031 & 0.0208 \\
0.15 & 0.3509 & 0.2930 & 0.1880 & 0.0946 & 0.0116 \\
0.2 & 0.3477 & 0.2867 & 0.1791 & 0.0855 & 0.0019 \\
0.25 & 0.3439 & 0.2806 & 0.1705 & 0.0757 & 0.0110 \\
\hline
\end{tabular}


Table 5. Maximum allowable upper bounds $b$ of Example 1.

\begin{tabular}{cccccc}
\hline $\boldsymbol{e}$ & $\boldsymbol{\alpha}=\mathbf{0}$ & $\boldsymbol{\alpha}=\mathbf{0 . 1}$ & $\boldsymbol{\alpha}=\mathbf{0 . 1 5}$ & $\boldsymbol{\alpha}=\mathbf{0 . 2}$ & $\boldsymbol{\alpha}=\mathbf{0 . 2 5}$ \\
\hline 0.05 & 0.3358 & 0.2292 & 0.1825 & 0.1401 & 0.1022 \\
0.1 & 0.3181 & 0.2129 & 0.1670 & 0.1253 & 0.0883 \\
0.15 & 0.3005 & 0.1965 & 0.1513 & 0.1106 & 0.0744 \\
0.2 & 0.2828 & 0.1802 & 0.1357 & 0.1019 & 0.0605 \\
0.25 & 0.2651 & 0.1638 & 0.1201 & 0.0820 & 0.0466 \\
\hline
\end{tabular}

Table 6. Maximum allowable upper bounds $\alpha$ of Example 1.

\begin{tabular}{cccccc}
\hline $\boldsymbol{e}$ & $\boldsymbol{b}=\mathbf{0 . 0 5}$ & $\boldsymbol{b}=\mathbf{0 . 1}$ & $\boldsymbol{b}=\mathbf{0 . 1 5}$ & $\boldsymbol{b}=\mathbf{0 . 2}$ & $\boldsymbol{b}=\mathbf{0 . 2 5}$ \\
\hline 0.05 & 0.3317 & 0.2531 & 0.1879 & 0.1307 & 0.0792 \\
0.1 & 0.3099 & 0.2335 & 0.1697 & 0.1135 & 0.0629 \\
0.15 & 0.2881 & 0.2150 & 0.1515 & 0.0964 & 0.0464 \\
0.2 & 0.2664 & 0.2010 & 0.1334 & 0.0792 & 0.0301 \\
0.25 & 0.2447 & 0.1749 & 0.1154 & 0.0622 & 0.0138 \\
\hline
\end{tabular}

Table 7. Maximum allowable upper bounds $b$ of Example 1.

\begin{tabular}{cccccc}
\hline$f$ & $\boldsymbol{\alpha}=\mathbf{0}$ & $\boldsymbol{\alpha}=\mathbf{0 . 1}$ & $\boldsymbol{\alpha}=\mathbf{0 . 1 5}$ & $\boldsymbol{\alpha}=\mathbf{0 . 2}$ & $\boldsymbol{\alpha}=\mathbf{0 . 2 5}$ \\
\hline 0.05 & 0.3712 & 0.2619 & 0.2137 & 0.1697 & 0.1310 \\
0.1 & 0.3536 & 0.2456 & 0.1981 & 0.1549 & 0.1161 \\
0.15 & 0.3358 & 0.2292 & 0.1825 & 0.1401 & 0.1022 \\
0.2 & 0.3181 & 0.2129 & 0.1669 & 0.1253 & 0.0883 \\
0.25 & 0.3005 & 0.1965 & 0.1513 & 0.1106 & 0.0745 \\
\hline
\end{tabular}

Table 8. Maximum allowable upper bounds $\alpha$ of Example 1.

\begin{tabular}{cccccc}
\hline $\boldsymbol{f}$ & $\boldsymbol{b}=\mathbf{0 . 0 5}$ & $\boldsymbol{b}=\mathbf{0 . 1}$ & $\boldsymbol{b}=\mathbf{0 . 1 5}$ & $\boldsymbol{b}=\mathbf{0 . 2}$ & $\boldsymbol{b}=\mathbf{0 . 2 5}$ \\
\hline 0.05 & 0.3748 & 0.2930 & 0.2242 & 0.1651 & 0.1130 \\
0.1 & 0.3534 & 0.2726 & 0.2061 & 0.1479 & 0.1010 \\
0.15 & 0.3317 & 0.2531 & 0.1879 & 0.1307 & 0.0792 \\
0.2 & 0.3099 & 0.2335 & 0.1697 & 0.1135 & 0.0629 \\
0.25 & 0.2881 & 0.2150 & 0.1515 & 0.0964 & 0.0464 \\
\hline
\end{tabular}

Example 2. Consider the equation focused on in $[14,15]$ as follows:

$$
\frac{d}{d t}[x(t)+0.2 x(t-\tau(t))]+0.6 x(t)-0.5 \tanh x(t-\sigma(t))=0, \quad t \geq 0,
$$

when $\sigma_{2}=0.2$. and $\tau(t)=\frac{\cos ^{2}(t)}{10}$.

Solving our criterion (23) for guaranteeing uniformly-exponential stability, $\alpha=0.0038$ is given, then we get the maximum allowable upper bound of the delay $\sigma=9.00$. Table 9 presents the comparison of the maximum allowable value of $\sigma$ obtained in Corollary 1 . We can see that our results are much better than those obtained in $[14,15]$.

Table 9. Maximum allowable upper bounds $\sigma$ for Example 2.

\begin{tabular}{ccc}
\hline Methods & $\boldsymbol{\alpha}=\mathbf{0 . 0 0 3 8}$ & $\boldsymbol{\alpha}=\mathbf{0 . 0 2 8}$ \\
\hline H.Chen and X. Meng [15] & infeasible & infeasible \\
P. Keadnarmol and T. Rojsiraphisal [14] & 7.5231 & 0.0321 \\
Our result (23) & 9.00 & 0.1850 \\
\hline
\end{tabular}


Example 3. Consider the neutral differential equation focused on in $[1,9,14,15]$ as follows:

$$
\frac{d}{d t}[x(t)+0.2 x(t-0.1)]+0.6 x(t)-0.3 \tanh x(t-\sigma)=0, \quad t \geq 0 .
$$

Solving our criterion (23) to guarantee uniformly-exponential stability, $\alpha=0.0038$ is given, then we get the maximum allowable upper bound of the delay $\sigma=175.3543$. Table 10 presents the comparison of the maximum allowable value of $\sigma$ obtained in Corollary 1. We can see that our results are much better than the other works.

Table 10. Maximum allowable upper bounds $\sigma$ of Example 3.

\begin{tabular}{cc}
\hline Methods & $\boldsymbol{\alpha}=\mathbf{0 . 0 0 3 8}$ \\
\hline H. Chen [1] & 175.2890 \\
H. Chen and X. Meng [15] & $10^{21}(\mathrm{No} \alpha)$ \\
T. Rojsiraphisal and P. Niamsup [9] & 1.9470 \\
P. Keadnarmol and T. Rojsiraphisal [14] & 175.3540 \\
Our result (23) & 175.3543 \\
\hline
\end{tabular}

\section{Conclusions}

We improved the criteria for the exponential stability of a new certain nonlinear neutral differential equation with a more generally interval-distributed and discrete time-varying delays. By using the Lyapunov-Krasovkii functional, descriptor model transformation, the Leibniz-Newton formula, and utilization of the zero equation, the exponential stability criteria were in the form of linear matrix inequalities (LMIs). Finally, we presented numerical examples to show the effectiveness of the theoretical results and to illustrate less conservative conditions than many other works.

Author Contributions: The authors claim to have contributed significantly and equally to this work. All authors read and approved the final manuscript.

Funding: The second author was financially supported by the National Research Council of Thailand, Khon Kaen University 2019. The third author was supported by the Thailand Research Fund (TRF), the Office of the Higher Education Commission (OHEC) (Grant Number MRG6080061) and the University of Phayao, Phayao, Thailand 2019.

Acknowledgments: The authors thank the reviewers for their valuable comments and suggestions, which led to the improvement of the content of the paper.

Conflicts of Interest: The authors declare no conflict of interest.

\section{References}

1. Chen, H. Some Improved Criteria on Exponential Stability of Neutral Differential Equation. Adv. Differ. Equ. 2012, 2012, 170. [CrossRef]

2. El-Morshedy, H.A.; Gopalsamy, K. Nonoscillation, oscillation and convergence of a class of neutral equations. Nonlinear Anal. 2000, 40, 173-183. [CrossRef]

3. Kwon, O.M.; Park, J.H. On improved delay-dependent stability criterion of certain neutral differential equations. Appl. Math. Comput. 2008, 199, 385-391. [CrossRef]

4. Li, X. Global exponential stability for a class of neural networks. Appl. Math. Lett. 2009, 22, 1235-1239. [CrossRef]

5. Nam, P.T.; Phat, V.N. An improved stability criterion for a class of neutral differential equations. Appl. Math. Lett. 2009, 22, 31-35. [CrossRef]

6. Park, J.H. Delay-dependent criterion for asymptotic stability of a class of neutral equations. Appl. Math. Lett. 2004, 17, 1203-1206. [CrossRef]

7. Park, J.H.; Kwon, O.M. Stability analysis of certain nonlinear differential equation. Chaos Solitons Fractals 2008, 37, 450-453. [CrossRef]

8. Sun, Y.G.; Wang, L. Note on asymptotic stability of a class of neutral differential equations. Appl. Math. Lett. 2006, 19, 949-953. [CrossRef]

9. Rojsiraphisal, T.; Niamsup, P. Exponential stability of certain neutral differential equations. Appl. Math. Comput. 2010, 217, 3875-3880. [CrossRef] 
10. Gopalsamy, K.; He, X.Z. Stability in asymmetric Hopfield nets with transmission delays. Phys. D Nonlinear Phenom. 1994, 76, 344-358. [CrossRef]

11. Gopalsamy, K.; Leung, I. Delay induced periodicity in a neural netlet of excitation inhibition. Phys. D Nonlinear Phenom. 1996, 89, 395-426. [CrossRef]

12. Gopalsamy, K.; Leung, I. Convergence under dynamical thresholds with delays. IEEE Trans. Neural Netw. 1997, 8, 341-348. [CrossRef] [PubMed]

13. Gopalsamy, K.; Leung, I.; Liu, P. Global Hopf-bifurcation in a neural netlet. Appl. Math. Comput. 1998, 94, 171-192. [CrossRef]

14. Keadnarmol, P.; Rojsiraphisal, T. Globally exponential stability of a certain neutral differential equation with time-varying delays. Adv. Differ. Equ. 2014, 2014, 32. [CrossRef]

15. Chen, H.; Meng, X. An Improved Exponential Stability Criterion for a class of neutral delayed differential equations. Appl. Math. Lett. 2011, 24, 1763-1767. [CrossRef]

16. Chatbupapan, W.; Mukdasai, K. New delay-range-dependent exponential stability criteria for certain neutral differential equations with interval discrete and distributed time-varying delay. Adv. Differ. Equ. 2016, 2016, 324. [CrossRef]

17. Beretta, E.; Breda, D. Discrete or distributed delay? Effects on stability of population growth. Math. Biosci. Eng. 2016, 13, 19-41. [CrossRef] [PubMed]

18. Liu, Q.; Jiang, D. Stationary distribution and extinction of a stochastic predator-prey model with distributed delay. Appl. Math. Lett. 2018, 78, 79-87. [CrossRef]

19. Xu, C.; Shao, Y. Bifurcations in a predator-prey model with discrete and distributed time delay. Nonlinear Dyn. 2012, 63, 2207-2223. [CrossRef]

20. Karmeshu, V.; Gupta, K.; Kadambari, V. Neuronal model with distributed delay: Analysis and simulation study for gamma distribution memory kernel. Biol. Cybern. 2011, 104, 369-383. [CrossRef]

21. Sharma, S.K. Neuronal model with distributed delay: Emergence of unimodal and bimodal ISI distributions. IEEE Trans. Nanobiosci. 2013, 12, 1-12. [CrossRef]

22. Maharajan, C.; Raja, R.; Cao, J.; Rajchakit, G. Novel global robust exponential stability criterion for uncertain inertial-type BAM neural networks with discrete and distributed time-varying delays via Lagrange sense. J. Frankl. Inst. 2018, 355, 4727-4754. [CrossRef]

23. Maharajan, C.; Raja, R.; Cao, J.; Rajchakit, G.; Alsaedi, A. Novel results on passivity and exponential passivity for multiple discrete delayed neutral-type neural networks with leakage and distributed time-delays. Chaos Solitons Fractals 2018, 115, 268-282. [CrossRef]

24. Samidurai, R.; Rajavel, S.; Zhu, Q.; Raja, R.; Zhou, H. Robust passivity analysis for neutral-type neural networks with mixed and leakage delays. Neurocomputing 2016, 175, 635-643. [CrossRef]

25. Zhu, Q.; Senthilraj, S.; Raja, R.; Samidurai, R. Stability analysis of uncertain neutral systems with discrete and distributed delays via the delay partition approach. Int. J. Control Autom. Syst. 2017, 15, 2149-2160. [CrossRef]

26. Khongja, N.; Botmart, T.; Niamsup, P.; Weera, W. Guaranteed cost control for exponential stability of a nonlinear system with mixed time-varying delays in states and controls. Adv. Differ. Equ. 2018, 2018, 435. [CrossRef]

27. Agarwal, R.P.; Grace, S.R. Asymptotic Stability of Certain Neutral Differential Equations. Math. Comput. Model. Dyn. Syst. 2000, 31, 9-15. [CrossRef]

28. El-Metwally, H.; Kulenovi, M.R.S.; Hadziomerspahi, S. Nonoscillatory solutions for system of neutral delay equation. Nonlinear Anal. 2003, 54, 63-81. [CrossRef]

29. Kulenovi, M.R.S.; Hadziomerspahi, S. Existence of nonoscillatory solution of second order linear neutral delay equation. J. Math. Anal. Appl. 1998, 228, 436-448. [CrossRef]

30. Kulenovic, M.R.S.; Ladas, G.; Meimaridou, A. Necessary and sufficient conditions for oscillations of neutral differential equations. J. Aust. Math. Soc. Ser. 1987, 28, 362-375. [CrossRef]

31. Gu, K.; Kharitonov, V.L.; Chen, J. Stability of Time-Delay Systems; Birkhäuser: Berlin, Germany, 2003.

32. Peng, C.; Fei, M.R. An Improved Result on the Stability of Uncertain T-S Fuzzy Systems with Interval Time-Varying Delay. Fuzzy Sets Syst. 2013, 212, 97-109. [CrossRef] 
(C) 2019 by the authors. Licensee MDPI, Basel, Switzerland. This article is an open access article distributed under the terms and conditions of the Creative Commons Attribution (CC BY) license (http:/ / creativecommons.org/licenses/by/4.0/). 